\title{
CRITÉRIOS DE SEGURANÇA DA MOBILIZAÇÃo PRECOCE DE PACIENTES CRÍTICOS NA UTI
}

\section{ARTIGO ORIGINAL}

SOUSA, Clorismar Bezerra de ${ }^{1}$

ALMEIDA, Leandro Augusto ${ }^{2}$

SOUSA, Clorismar Bezerra de. ALMEIDA, Leandro Augusto. Critérios de segurança da mobilização precoce de pacientes críticos na UTI. Revista Científica Multidisciplinar Núcleo do Conhecimento. Ano 05, Ed. 06, Vol. 09, pp. 74-82. Junho de 2020. ISSN: 2448-0959, Link de acesso: https://www.nucleodoconhecimento.com.br/saude/criterios-de-seguranca

\section{RESUMO}

Atualmente aumentou o índice de morbidades causadas pelo imobilismo dos pacientes internados nas Unidades de Terapia Intensiva (UTI). Em consequência disto, os custos assistenciais aumentaram e reduziu da sobrevida após alta hospitalar. Para evitar esses agravos surgem à mobilização precoce (MP), uma intervenção viável e segura capaz de promover a preservação da funcionalidade e recuperação do paciente. O objetivo deste trabalho é apresentar os critérios de segurança da mobilização precoce de pacientes internados na Unidade de Terapia Intensiva. (UTI). Trata-se de uma revisão bibliográfica, narrativa, exploratória, de abordagem qualitativa, baseada em dados eletrônicos: PuBMed, LILACS, SciELO, Google acadêmico, em língua portuguesa e inglesa. Obtivemos 62 artigos relacionados com o tema proposto. O repouso prolongado causa efeitos deletérios como úlceras de

\footnotetext{
${ }^{1}$ Graduada em Fisioterapia e pós graduando em Terapia Intensiva pelo IEES.

${ }^{2}$ Mestrando em Bioengenharia pela Universidade Brasil com defesa dia 25 de junho de 2020, Especialista em UTI neonatal pela Unicamp, Especialista em Fisioterapia Intensiva pela ASSOBRAFIR.
} 
pressão, disfunções da funcionalidade, complicações hemodinâmicas, neurológicas e cardíacas podendo se agravar pelo uso de medicamentos e sedativos. A reabilitação precoce do paciente deve iniciar logo após sua estabilização clínica podendo ser realizada mesmo na fase de sedação ou coma. Para que ocorra de forma segura, é indispensável a avaliação dos critérios de segurança: cardiovascular, neurológica e respiratória para que a conduta terapêutica não agrave o quadro clínico do paciente. Conclui-se ao fim dos estudos que essa conduta terapêutica está diretamente ligada à melhora física e psicológica do indivíduo e que o fisioterapeuta dentro da Unidade de Terapia Intensiva é uma base e não apenas um complemento na qual suas condutas reduzem ou eliminam os efeitos provindos da inatividade.

Palavras-chave: Fisioterapia, cuidados críticos, reabilitação precoce.

\section{INTRODUÇÃO}

$\mathrm{Na}$ unidade de terapia intensiva (UTI) é cada vez mais frequente os pacientes permanecerem restritos ao leito, causando disfunção severa do sistema osteomioarticular, inatividade, descondicionamento físico e fraqueza muscular. (FEITOZA et al., 2014). Atualmente teve um alto índice das morbidades causadas pela permanência prolongada no leito e imobilidade dos pacientes internados nestas unidades, (NOAL et al., 2019) em paralelo tornaram-se maior os custos assistenciais, prejuízos à qualidade de vida, mortalidade e redução da sobrevida após alta hospitalar. (FERREIRA et al., 2019).

O repouso prolongado causam efeitos deletérios como úlceras de pressão, disfunções da funcionalidade, diminuição da massa muscular, complicações hemodinâmicas, neurológicas e cardíacas, aumento do tempo de internação, podendo se agravar pelo uso de medicamentos e sedativos. (HOSTEIN; CASTRO, 2019). Esses pacientes das UTIs apresentam grande risco de desenvolver a "síndrome do desuso" em decorrência da própria patologia ou imobilização terapêutica. (CAZEIRO; PERES, 2010).

A mobilização precoce (MP) tem surgido como uma intervenção viável e segura capaz de promover a preservação da funcionalidade e recuperação do paciente. (MACHADO 
et al., 2016). Segundo as Diretrizes Brasileiras de Ventilação Mecânica em 2013 a MP deve ser iniciada em menos de 72 horas após início da ventilação mecânica (VM) para que alcance resultados funcionais significantes. (NYDAHL et al., 2014). Stiller e Philips foram os primeiros autores a dar informações sobre os critérios de segurança utilizados para realizar a MP nas UTIs. Os estudos foram fundamentados nas experiências clínicas dos autores e nos princípios fisiológicos. Recomendou-se que a técnica seja executada com segurança e monitorização apropriada e realizada de forma segura e eficiente, onde os desfechos sejam favoráveis ao paciente. (CONCEIÇÃO et al., 2017).

A mobilização precoce necessita de mais estudos que evidenciam a sua aplicabilidade, contribuindo assim para incremento do conhecimento científico sobre este tema. Esta revisão de literatura tem como objetivo apresentar os critérios de segurança da Mobilização Precoce em pacientes críticos das Unidades de Terapia Intensiva, onde se faz necessário o reconhecimento desses critérios a fim de proporcionar ao paciente uma resposta benéfica proveniente desta abordagem.

\section{MATERIAIS E MÉTODOS}

Este estudo trata-se de uma revisão bibliográfica, narrativa, exploratória, de abordagem qualitativa, baseada em dados eletrônicos: PuBMed, LILACS, SciELO, Google acadêmico, em língua portuguesa e inglesa, além de produções intelectuais como teses, dissertações e monografias retiradas de repositórios institucionais de universidades públicas do Brasil. Tivemos como critérios de inclusão estudos publicados no período de 2010 a 2020 que busca demonstrar os critérios de segurança da Mobilização Precoce em pacientes críticos da Unidade de Terapia Intensiva. Foram excluídos materiais que não contemplem o período supracitado, resumos, reportagens e demais publicações que não tenham cunho científico. A busca dos dados foi realizada no período de fevereiro a março do ano de 2020. Foram utilizadas para busca dos materiais, as palavras - chave: Fisioterapia, Cuidados críticos e Reabilitação precoce. Com o auxílio dos descritores foram encontrados 62 artigos relacionados com o tema proposto. 


\section{REVISÃO DE LITERATURA}

A partir do ano de 1860 foi empregado de forma abusiva o repouso no leito como uma prática terapêutica, pois havia um mito que era bom para o doente. (CAZEIRO; PERES, 2010) e era regularmente prescrito pela equipe médica no meio da terapia intensiva, pois confiava que essa estabilização clínica era eficaz ao paciente. Com o crescimento dos conhecimentos científicos e tecnológicos dos estudos na área, confirmou-se que a imobilidade no leito aumenta significativamente o tempo de Ventilação Mecânica (VM), (HOSTEIN; CASTRO, 2019) acarreta comprometimentos hemodinâmicos e físicos, onde a maioria dos pacientes apresentam alterações neuromusculares e motoras interferindo na independência das atividades da vida diária, levando a um declínio funcional e prejudicando assim as funções vitais deste paciente. (SILVA; OLIVEIRA, 2015).

A imobilidade é um problema frequente que contribui no surgimento da fraqueza na musculatura respiratória e periférica. (FELICIANO et al., 2012) Essa perda de massa muscular varia de $3 \%$ a $11 \%$ nas primeiras 3 semanas de imobilização. (Ferreira et al., 2019). A incidência de perdas de fibras musculares é de aproximadamente de $30 \%$ a $60 \%$ de pacientes internados nas UTIs. Após a II Guerra Mundial os autores Corcoran, Halar, Bell, Leduc e Rousseau, realizaram estudos e observaram que a mobilização precoce (MP) de pessoas lesionadas geravam benefícios. (CAZEIRO; PERES, 2010).

Para evitar os efeitos deletérios do imobilismo, surgem à mobilização precoce (MP). Conceituado como reabilitação do paciente crítico com início imediato após estabilização clínica do paciente, podendo ser realizada mesmo na fase de sedação ou coma, onde o principal objetivo é proporcionar movimento ao paciente diminuindo sua imobilização. Neste sentido a MP deve ser realizada de forma segura, viável e eficaz, com os critérios de segurança bem avaliados antes da terapia, para que tenha certeza de que esta conduta terá benefícios significativos ao paciente. (SANTOS; BORGES, 2020). 
Essa conduta terapêutica atua promovendo benefícios na função pulmonar, cardíaca e muscular diminuindo o esforço respiratório, aumentando assim os volumes pulmonares, auxiliando no transporte de oxigênio e promovendo ganho de força muscular. A fisioterapia apresentou resultados positivos atuando não somente na reabilitação, mas no processo preventivo de complicações que venham surgir para o paciente o qual se apresenta frágil. (SANTOS; BORGES, 2020). Brito et al. (2015) afirma ainda que a MP é muito eficaz tanto ao paciente quanto na redução de custos hospitalares.

O critério de segurança é se certificar que o paciente se encontra com estabilidade hemodinâmica, mesmo que inconsciente. Nos pacientes que apresentam em coma ou sedados, as intervenções tem início com mobilizações articulares passivas para prevenir encurtamento muscular, úlceras de pressão e manter amplitude de movimento. (HOSTEIN; CASTRO, 2019). A evolução para exercícios ativos depende do estado de alerta do paciente, grau de força muscular e de apresentar hemodinamicamente estável, evoluindo progressivamente para exercícios ativoassistido, ativo-livres e ativo-resistidos, onde a intensidade depende de acordo com a progressão e evolução do paciente. (HOSTEIN; CASTRO, 2018)

Quanto aos fatores neurológicos o paciente não pode apresentar aumento da pressão intracraniana, agitação, e precisa entender e obedecer a comandos verbais adequados, como abrir os olhos. Já na parte cardiovascular, os critérios de segurança são: $F C>40$ bpm e < 130 bpm; pressão arterial sistólica (PAS) $>90$ mmhg e $<180$ mmhg; e pressão arterial média (PAM) $>60 \mathrm{mmhg}$ e $<110 \mathrm{mmhg}$. (AQUIM et al., 2019). Quanto à avaliação do nível de consciência dos doentes, recomenda-se diminuir ou interromper as sedações diárias para que essa avaliação tenha resultado mais fidedigno, além de diminuir o grau de complicação relacionadas a internação do paciente. (CONCEIÇÃO et al., 2017).

Sendo assim a MP atribui de inúmeras particularidades no que diz respeito à ação, e contém várias formas de aplicabilidade que varia de acordo com a necessidade do paciente. É indispensável realizar uma avaliação antes da conduta para que tenha eficácia nos desfechos clínicos deste doente. (AZEVEDO; GOMES, 2015). Mas para 
que ocorra de forma segura é indispensável à avaliação dos critérios de segurança: cardiovascular, neurológica e respiratória para que a MP não agrave o quadro clínico do paciente. (SARTI et al., 2016).

Estudos comprovam que essa conduta terapêutica está diretamente ligada à melhora física e psicológica do indivíduo, interferindo de forma significativa o tempo de permanência hospitalar e do uso de VM, que é bastante comum nas UTIs. O protocolo desta terapia envolve desde o exercício passivo até a posição ortostática acompanhada da deambulação, melhorando alterações funcionais dos vários sistemas do corpo humano, como no sistema imunológico que aumenta a produção de citocinas anti inflamatórias reduzindo o stress oxidativo e as inflamações. (PINTO et al., 2018).

A MP dentro da Unidade de Terapia Intensiva tem a função de tornar o trabalho da fisioterapia de grande importância, pois tal função não é somente garantir a sobrevida, mas, sobretudo oferecer qualidade de vida. (SANTOS; BORGES, 2020). McWillians et.al., afirmam que o fisioterapeuta é essencial na equipe multidisciplinar intervindo precocemente nos pacientes críticos promovendo aumento significativo na mobilidade desses pacientes na alta da UTI. Em outro estudo Lord et al., confirmam que um protocolo de MP reduz em até $21,8 \%$ o tempo de internação dos pacientes graves. (COSTA et al., 2019).

Marina Melo Coelho realizou um estudo clínico experimental na Unidade de Terapia Intensiva Adulto do Hospital de Clínicas da Universidade Federal de Uberlândia. Neste estudo foram incluídos 33 pacientes, mas por motivos de exclusão e alguns óbitos restaram apenas 10. Formaram dois grupos: 05 no grupo controle (GC) que receberam fisioterapia convencional e 05 no grupo intervenção (Gl) que participaram de um protocolo de mobilização precoce. Após estudo conclui-se que a utilização dos protocolos de mobilização precoce foi eficaz para melhorar a qualidade de vida desses pacientes. (COELHO, 2019).

Em um estudo apresentado por Thaís Borgheti de Figueiredo, á Faculdade de Medicina da Universidade de São Paulo, para obtenção de título de mestre em 
Ciências, onde realizaram mobilização passiva e exercícios ativos no leito em 74 pacientes admitidos na Unidade de Terapia Intensiva do Hospital das Clínicas de São Paulo. Conclui-se ao final do estudo que a fisioterapia motora em uma UTI de queimados também é viável e segura. (FIGUEIREDO, 2019).

Debora Stripari Schujmann Nogueira desenvolveu um estudo prospectivo observacional com 62 pacientes da Unidade de Terapia Intensiva do Hospital das Clínicas de São Paulo. Os critérios de segurança eram ser maior de 18 anos, sem diagnósticos neurológicos e sem contra indicações para mobilização. Através deste estudo mostrou-se que atividades leves foram consideradas um fator de proteção para os pacientes na Unidade de Terapia Intensiva. (SCHUJMANN, 2016).

Elisângela Pinto Marinho de Almeida, 2016 desenvolveu uma tese para obtenção do título de Doutor em Ciências. Foi realizado um estudo unicentrico, randomizado e controlado, onde se fizeram presentes 108 pacientes que foram submetidos à cirurgia oncológica abdominal de grande porte. Divididos em Grupo Intervenção (Gl) que foram submetidos a um protocolo de mobilização precoce e Grupo Controle (GC) que participaram de uma reabilitação convencional. No fim do estudo concluíram que os pacientes do Gl obtiveram redução da perda funcional quando comparados ao GC. (ALMEIDA, 2016).

As condutas fisioterapêuticas têm se mostrado bastante eficaz na preservação e recuperação da funcionalidade do paciente, onde o trabalho desse profissional diminui as sequelas causadas pela hospitalização, oferecendo ao paciente uma melhor qualidade de vida. (MONDADORI et al., 2016).

\section{CONCLUSÃO}

Foi possível observar nesta revisão de literatura que quando se respeita todos os critérios de segurança da mobilização precoce se alcança resultados positivos nos desfechos clínicos do paciente crítico. Concluindo que o protocolo de MP é considerado uma conduta viável, eficaz e segura que reduz significativamente as incidências de complicações causadas pela imobilidade, diminui o tempo de 
ventilação mecânica e o tempo de hospitalização, diminuindo taxa de mortalidade, oferecendo ao paciente uma melhor qualidade de vida.

O fisioterapeuta dentro da Unidade de Terapia Intensiva é uma base e não apenas um complemento na qual suas condutas reduzem ou eliminam os efeitos provindos da inatividade, mantendo ou devolvendo as funções vitais dos sistemas corporais, reduzindo as chances de complicações clínicas.

\section{REFERÊNCIA}

ALMEIDA, E. P. M. Efeito de um protocolo pós-operatório de mobilização precoce na recuperação funcional e nas complicações clínicas pós- operatórias de pacientes submetidos a cirurgia oncológica abdominal de grande porte. Tese. Faculdade de Medicina da Universidade de São Paulo, São Paulo, 2016.

AQUIM, et al. Diretrizes Brasileiras de Mobilização Precoce em Unidade de Terapia Intensiva. Rev Bras Ter Intensiva, n. 4, v. 31, p. 434-443, set, 2019.

ARAÚJO, et al. A influência da mobilização precoce no tempo de internamento na Unidade de Terapia Intensiva. ASSOBRAFIR Ciência, n. 2, v. 3, p. 31-42, agos, 2012.

AZEVEDO, P. M. D. S.; GOMES, B. P. Efeitos da mobilização precoce na reabilitação funcional em doentes críticos: uma revisão sistemática. Revista de Enfermagem Referência, n. 5, v. 4, p. 129-138, jun, 2015.

BRITO, M. C. S.; SILVA, L. W.; RIBEIRO, E. Mobilização precoce em pacientes adultos submetidos à ventilação mecânica (VM) na unidade de terapia intensiva (UTI). Rev. Eletrôn. Atualiza Saúde, n. 2, v.2, dez, 2015.

CAZEIRO, A. P. M.; PERES, P. T. A terapia ocupacional na prevenção e no tratamento de complicações decorrentes da imobilização no leito. Cadernos de Terapia Ocupacional da UFSCar, n. 2, v. 18, p. 149-167, agos, 2010. 
COELHO, M. M. Impacto da mobilização precoce na qualidade de vida de sobreviventes de sepse. Dissertação. Universidade Federal de Uberlândia, Uberlândia, 2019.

CONCEIÇAO, et al. Critérios de segurança para iniciar a mobilização precoce em unidades de terapia intensiva. Revisão sistemática. Rev Bras Ter Intensiva, n.4, v. 29, p. 509-519, abril, 2017.

COSTA, et al. Avaliação de um protocolo de mobilização precoce em uma Unidade de Terapia Intensiva. Revista Conhecimento Online, v. 3, dez, 2019.

FEITOSA, C. L.; JESUS, P. K. S.; NOVAIS, R. O.; GARDENGHI, G. Eficácia da fisioterapia motora em unidades de terapia intensiva, com ênfase na mobilização precoce. Revista Eletrônica Saúde e Ciência, n. 1, v. 4, abril, 2014.

FERREIRA, et al. Segurança e potenciais benefícios da fisioterapia em adultos submetidos ao suporte de vida com oxigenação por membrana extracorpórea: uma revisão sistemática. Rev. Bras Ter Intensiva, n. 2, v. 31, p. 227-239, set, 2019.

HOLSTEIN, J. M.; CASTRO, A. A. M. Benefícios e métodos da mobilização precoce em uti: uma revisão sistemática. Life Style Journal, n. 2, v. 6, p. 07-22, ago, 2019.

HOLSTEIN, J. M.; CASTRO, A. A. M. Protocolos de mobilização precoce: nossas equipes estão preparadas para a implementação? Salão internacional de ensino, pesquisa e extensão - SIEPE, Nov, 2018.

MACHADO, A. S.; NUNES, R. D.; REZENDE, A. A. B. Intervenções fisioterapêuticas para mobilizar precocemente os pacientes internados em Unidades de Terapia Intensiva: estudo de revisão. Revista Amazônia Sciene \& Health, n. 2, v. 5, p. 41 46, abr, 2016.

MONDADORI, et al. Humanização da fisioterapia em Unidade de Terapia Intensiva Adulto: estudo transversal. Fisioter Pesqui., n. 3, V. 23, p. 294-300, 2016. 
NOAL, S.; GUEDES, P. F.; COSTENARO, R. G. S. Benefícios da mobilização precoce em pacientes internados em uma unidade de terapia intensiva. Disciplinarum Scientia, n. 2, v. 20, p. 447- 457, set, 2019.

NOGUEIRA, D. S. S. Impacto do nível de atividade física na funcionalidade de pacientes na unidade de terapia intensiva. Dissertação. Universidade de São Paulo, São Paulo, 2016.

NYDAHL, P. et al. Early Mobilization of Mechanically Ventilated Patients: A 1-Day Point-Prevalence Study in Germany. Critical Care Medicine, n. 5, v. 42, p. 1178-1186, 2014.

PINTO, B. F.; PINTO, B. F.; DIAS, E. H. F. Efeitos sistêmicos da mobilização precoce em pacientes adultos internados na unidade de terapia intensiva: revisão atualizada. Fisioter Bras, n. 6, v. 19, p. 857-65, out, 2018.

SANTOS, J. S. S.; BORGES, A. R. A intervenção da fisioterapia na mobilização precoce em adultos dentro de uma unidade de terapia intensiva - UTI. Scientia Generalis, n. 2, v. 1, p. 11-22, 2020.

SARTI, T. C.; VECINA, M. V. A.; FERREIRA, P. S. N. Mobilização precoce em pacientes críticos. J Health Sci Inst., n, 3, v. 34, p. 177-82, 2016.

SILVA, I. T.; OLIVEIRA, A. A. Efeitos da mobilização precoce em pacientes críticos internados em UTI. C\&D- Revista Eletrônica da Fainor, n. 2, v. 8, p. 41-50, dez, 2015.

Enviado: Maio, 2020.

Aprovado: Junho, 2020. 